\title{
Anti-CD19 CAR T as a feasible and safe therapy for refractory/relapsed mixed phenotype acute leukemia (MPAL) patients
}

Xiangqun $\mathrm{Li}^{1}$, Kylann Chen ${ }^{2}$, Hailong Zhang ${ }^{1}$, Xian Zhang ${ }^{3}$, Junfang Yang ${ }^{3}$, Jing Liu ${ }^{1}$, Jianwei Zheng ${ }^{1}$, Fei Dong ${ }^{1}$, Yongbo Zhu ${ }^{1}$, Jiao $\mathrm{Yu}^{1}$, Hailong Zhang ${ }^{1}$, Peihua $\mathrm{Lu}^{3}$, and Bo Chen $^{1}$

${ }^{1}$ Kecellitics Biotech Company Ltd, Beijing, China

${ }^{2}$ University of Texas

${ }^{3}$ Hebei Yanda Lu Daopei Hospital

January 20, 2022

\begin{abstract}
Mixed phenotype acute leukemia (MPAL) is a relatively rare and heterogeneous group of diseases, which account for $2-5 \%$ of leukemia. So far, no optimal treatment regimens have been established for MPAL patients. CD19 chimeric antigen receptor $\mathrm{T}(\mathrm{CAR} \mathrm{T})$ therapy have shown remarkable efficacy in B-ALL patients. In this study, 5 MPAL patients received CD19 CAR $\mathrm{T}$ cell infusion and 3 out of 5 patients $(60 \%)$ achieved complete response (CR). 2 of 5 patients had only mild cytokine release syndrome (CRS). Neurotoxicity was not observed. Our data indicate that CD19 CAR T therapy is safe and effective for majority refractory/relapsed (r/r) MPAL patients.
\end{abstract}

Anti-CD19 CAR T as a feasible and safe therapy for refractory/relapsed mixed phenotype acute leukemia (MPAL) patients

Xiangqun Li $^{1}$ I Kylan Chen ${ }^{2}$ I Hailong Zhang ${ }^{1}$ I Xian Zhang ${ }^{3,4}$ I Junfang Yang ${ }^{3,4}$ I Jing Liu ${ }^{1}$ I Jianwei Zheng ${ }^{1}$ I Fei Dong ${ }^{1}$, Yongbo Zhu ${ }^{1}$ I Jiao Yu ${ }^{1}$ I Haihong Zhang ${ }^{1}$ I Peihua Lu $^{3,4}$ I Bo Chen ${ }^{1}$

${ }^{1}$ Kecellitics Biotech Company Ltd, Beijing, China

${ }^{2}$ College of Natural Sciences, University of Texas

${ }^{3}$ Lu Daopei Hospital, Langfang, Hebei, China ${ }^{4}$ Lu Daopei Institute of Hematology, Beijing, China

\section{Correspondence}

Peihua Lu, Lu Daopei Hospital, No. 6 Sipu Lang Road, Langfang, Hebei Province, China

Email: peihua_lu@126.com

Bo Chen, Kecellitics Biotech Company Ltd, No.88 Kechuang 6th Street, Beijing Economic Technological Developmental Zone, Beijing, China.

Email: bochen1008@outlook.com

ETHICAL APPROVAL 
This study was conducted according to the principles of the Declaration of Helsinki and with the approval of the Ethics Committee of Daopei Hospital.INFORMED CONSENT All the enrolled patients or their families provided informed consent.

AUTHOR CONTRIBUTIONS Peihua Lu, Bo Chen, and Xiangqun Li designed the clinical trial; Kylan Chen, Hailong Zhang, Xian Zhang, Junfang Yang, Jing Liu, Jianwei Zheng, Fei Dong, Yongbo Zhu, Jiao Yu, and Hailong Zhang executed the clinical trial and collected the data. Xiangqun Li analyzed the data and wrote the manuscript.

\section{CONFLICT of INTEREST}

All authors declare that they have no conflict of interest.

\section{DATA AVAILABILITY STATEMENT}

The data that support the findings of this study are available from the corresponding author upon reasonable request. CLINICAL TRIAL REGISTRATION NO: ChiCTR2000038532

Text word count: 1051 words

The number of figures: 1

The number of supplemental tables: 3

Abstract words: 104 words

To the Editor:

Mixed phenotype acute leukemia (MPAL) is a relatively rare and heterogeneous group of diseases, which account for $2-5 \%$ of leukemia. ${ }^{1}$ These cases are partitioned according to the lineage mix they display, i.e, as $\mathrm{B} /$ myeloid, $\mathrm{T} /$ myeloid, and rare types including triple lineage or $\mathrm{B} / \mathrm{T}$ co-expression. ${ }^{2} \mathrm{MPAL}$ is at higher risk of induction failure with a poor prognosis and an event-free survival (EFS) rate of less than $50 \%$ in children for the reason that mixed-phenotype leukemic stem cells are chemo-resistant owing to slow cell replication. ${ }^{3,4}$ So far, no optimal treatment regimens have been established for these patients. Despite CD19 chimeric antigen cell receptor (CAR T) therapy has demonstrated remarkable efficacy for B-cell malignancies, ${ }^{5,}{ }^{6}$ clinical benefit varies among different disease types. A previous study reported that B-ALL patients had a higher response rate $(93 \%)$ than CLL patients $(62 \%),{ }^{7}$ which indicates that even if CD19 expression is at a comparable level, the response sensitivity might be quite different. Nowadays, there is still no specific report on CAR T-cell therapy for refractory/relapsed (r/r) MPAL patients. Due to above considerations, we are interested to know whether CD19 CAR T cells can eradicate MPAL leukemia blasts and whether MAPL patients can benefit from CD19 CAR T cell therapy. Consequently, we carried out a study and reported the safety and efficacy of CD19 CAR T therapy for r/r MPAL patients (ChiCTR2000038532).

5 patients diagnosed as MPAL in 2019 were enrolled in this study. One patient was primary refractory disease and the other four patients had a second relapse. Patient 2, patient 3 and patient 5 displayed B/Myeloid bi-phenotype, patient 1 had a triple lineage (T/B/Myeloid) phenotype, whereas patient 4 had a T/Myeloid phenotype with CD19 aberrantly expression. In addition, patient 2 had the extramedullary disease (EMDs) with multiple sites, and patient 1 once previously underwent allogeneic hematopoietic stem cell transplantation (allo-HSCT). All patients had no central nervous system leukemia (CNSL) and all of them had bright CD19 expression on malignant B cells as examined by FCM. Detailed patient characteristics are summarized in Table S1.

Chemotherapy regimen before CAR cell infusion were listed in Table S1. Between May and September in 2019 , all the patients underwent fludarabine $\left(30 \mathrm{mg} / \mathrm{m}^{2}\right.$ per day $)$ and cyclophosphamide $\left(250 \mathrm{mg} / \mathrm{m}^{2}\right.$ per day) lymphodepleting chemotherapy for 3 consecutive days as described before. ${ }^{8}$ Subsequently, the patients received CAR T cells targeting CD19. The transduction efficiencies ranged from 12 to $67.5 \%$. The dose was $3^{*} 10^{5} / \mathrm{kg}$ of body weight. Dynamic CAR T expansion in peripheral blood (PB) was monitored at certain 
time intervals. All the patients underwent bone marrow (BM) biopsy examination and radiology studies on the day $30^{\text {th }}$ following the cell infusion to determine the response and remission status.

As shown in Figure 1A, CAR T cells showed an obvious expansion in 4 of 5 patients and peaked around day 10 or 11 in PB after infusion. With the expansion of CAR T cells, patient 1, patient 2 and patient 3 obtained FCM-MRD-negative CR on day 30 after infusion judged by flow cytometry. Wright-Giemsa staining also suggested that leukemia blasts disappeared in bone marrow on day 30 after infusion in patient 2 (Figure 1C)

Repeated PET/CT showed no evidence of abnormal FDG uptake compared to initial scans in patient 2 (Figure 1B), suggesting his multiple EMD lesions were also eradicated after CAR-T cell infusion. Notably, CAR $\mathrm{T}$ cells in patient 4 couldn't successfully expanded, which might be associated with her $\mathrm{T}$ cell's malignancies. She developed a serious pleural effusion on day 25 after CAR T infusion. MRD-FCM analysis showed that $94.09 \%$ of the nucleated cells in pleural effusion and $86.52 \%$ of the nucleated cells in the peripheral blood $(\mathrm{PB})$ were $\mathrm{CD} 7^{+} \mathrm{CD} 19^{-}$cells, which were almost malignant $\mathrm{T}$ lymphocytes, suggesting CAR T cells couldn't control leukemia progression. With the increase of the leukemia blasts burden, ascities, pericardiac effusion, pulmonary infection and septicemia also appeared one after another. Finally, this patient died on day 27 after CAR $\mathrm{T}$ infusion. Interestingly, although patient 5 had a remarkable expansion of CAR $\mathrm{T}$ cells, the leukemia blasts were non-responsive to the infusion. The reasons were unclear at this point.

As results, CR rate of $60 \%(3 / 5)$ was achieved among these 5 patients. Although the CR rate was seemingly lower than the previously reported CR rate in $\mathrm{r} / \mathrm{r}$ B-ALL ([?]90\%) patients, it is still significant for the $\mathrm{r} / \mathrm{r}$ MPAL patients, especially when there is no other optimal treatment available.

Meanwhile, we observed mild adverse events associated with associated with CD19 CAR T therapy. As shown in Figure 1D, with the expansion of CAR T cells, plasma IL10 and IFN $\gamma$ both elevated, which was consistent with our previous findings. ${ }^{9}$ Due to the mild cytokine release, patient 2 and patient 5 had grade 1 CRS manifested by fever, dizziness and slight fatigue, which was well managed with supportive care and dexamethasone. Neurotoxicity was not observed in any of these patients (Table S2). Serum CRP in patient 2 increased to $29.91 \mathrm{mg} / \mathrm{L}$ on day 9 after CAR T infusion, and G and GM experiments were positive, which all suggested fungi infection might have occurred. Accordingly, Lynaiamine and Bispycin B were given for anti-infection. No other adverse events were observed during the treatment.

Once achieved CR,patient 1, patient 2 and patient 3 were proceed to allo-HSCT on day 46, 59 and 53 after infusion, respectively (Table S3). Although the blasts were not completely eradicated, patient 5 still received allo-HSCT on day 48 after infusion. The 2-year Fellow-up data showed that patient 1 and patient 2 both remained CR till August 2021. Notably, although Patient 5 couldn't achieve CR after CD19 CAR T therapy, she achieved CR through all-HSCT and remained CR till the last follow-up. Unfortunately, patient 3 died of serious infection shock 5 months after allo-HSCT.

In summary, this study showed that despite the CR rate is lower compared with those B-ALL patients, the majority of refractory/relapsed MPAL patients with CD19 expression still can benefit from CD19 CAR T cell therapy. In addition, these patients maintain a better leukemia free survival (LFS) after bridging to HSCT following CAR T treatment, which raised a new therapeutic strategy to these $\mathrm{r} / \mathrm{r}$ MPAL patients. It should also be noted that only a small number of patients were enrolled in this study, more cases and further studies are needed to verify these findings.

\section{REFERENCTS}

1. Wolach O, Stone RM. Mixed-phenotype acute leukemia: current challenges in diagnosis and therapy. Curr Opin Hematol . 2017;24(2): 139-145.

2. Béné MC. Biphenotypic, bilineal, ambiguous or mixed lineage: strange leukemias! Haematologica . 2009;94(7): 891-893.

3. Lee HG, Baek HJ, Kim HS, Park SM, Hwang TJ, Kook H. Biphenotypic acute leukemia or acute leukemia of ambiguous lineage in childhood: clinical characteristics and outcome. Blood Res . 2019;54(1): 63-73. 
4. Brethon B, Lainey E, Caye-Eude A, Grain A, Fenneteau O, Yakouben K, et al. Case Report: Targeting 2 Antigens as a Promising Strategy in Mixed Phenotype Acute Leukemia: Combination of Blinatumomab With Gemtuzumab Ozogamicin in an Infant With a KMT2A-Rearranged Leukemia.Front Oncol . 2021;11: 637951.

5. Maude SL, Frey N, Shaw PA, Aplenc R, Barrett DM, Bunin NJ, et al. Chimeric antigen receptor T cells for sustained remissions in leukemia. N Engl J Med . 2014;371(16): 1507-1517.

6. Pan J, Yang JF, Deng BP, Zhao XJ, Zhang X, Lin YH, et al. High efficacy and safety of low-dose CD19directed CAR-T cell therapy in 51 refractory or relapsed B acute lymphoblastic leukemia patients.Leukemia . 2017;31(12): 2587-2593.

7. Cao JX, Gao WJ, You J, Wu LH, Liu JL, Wang ZX. The efficacy of anti-CD19 chimeric antigen receptor T cells for B-cell malignancies. Cytotherapy . 2019;21(7): 769-781.

8. Zhang X, Lu XA, Yang J, Zhang G, Li J, Song L, et al. Efficacy and safety of anti-CD19 CAR T-cell therapy in 110 patients with B-cell acute lymphoblastic leukemia with high-risk features. Blood Adv . 2020;4(10): 2325-2338.

9. Zhao Y, Liu Z, Wang X, Wu H, Zhang J, Yang J, et al. Treatment with Humanized Selective CD19CAR-T Cells Shows Efficacy in Highly Treated B-ALL Patients Who Have Relapsed after Receiving Murine-Based CD19CAR-T Therapies. Clin Cancer Res . 2019;25(18): 5595-5607.

\section{Figure legends}

FIGURE 1 CD19 CAR T cells are highly effective against MPAL patients.

(A) CAR T cell proliferation and persistence. Percentages of CD19 CAR T cells in peripheral blood were measured by flow cytometry before and after infusion. (B) FDG uptake in neck infiltrating loci disappeared after CAR T therapy. (C) Leukemia blasts in bone marrow aspirate before and post infusion were measured by Wright-Giemsa staining. (D) Cytokine levels in peripheral blood of patients were measured by ELISA assay at indicated time points after infusion. 
(A)

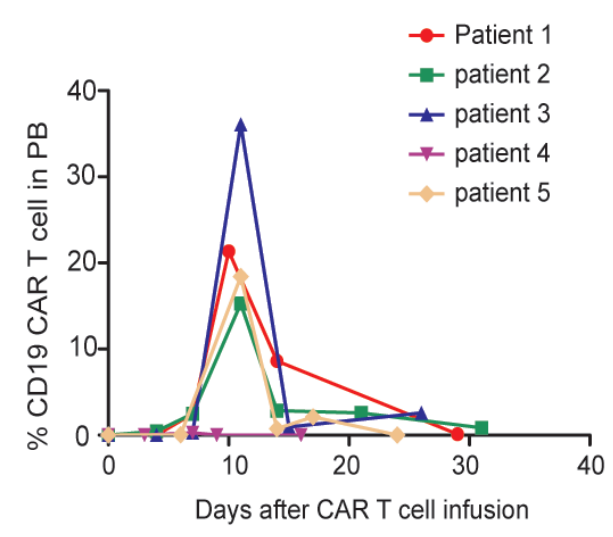

(C)

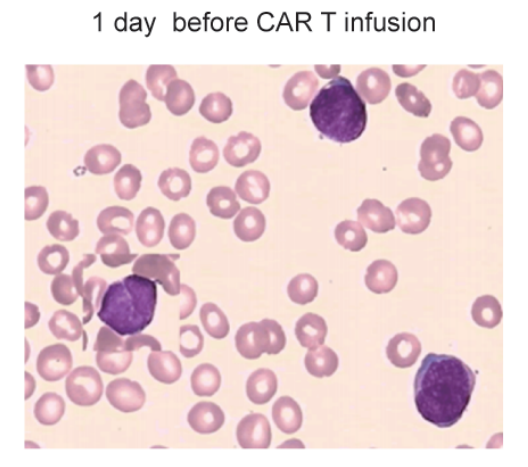

(B)
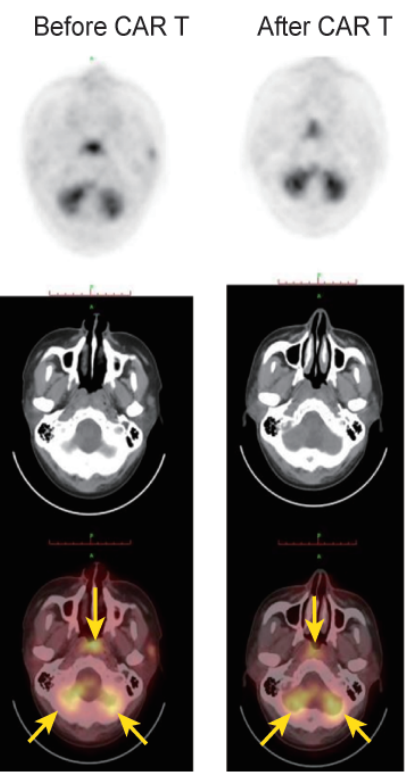

30 days after CAR T infusion

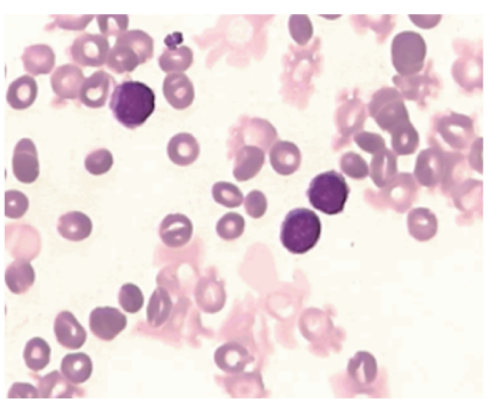

(D)

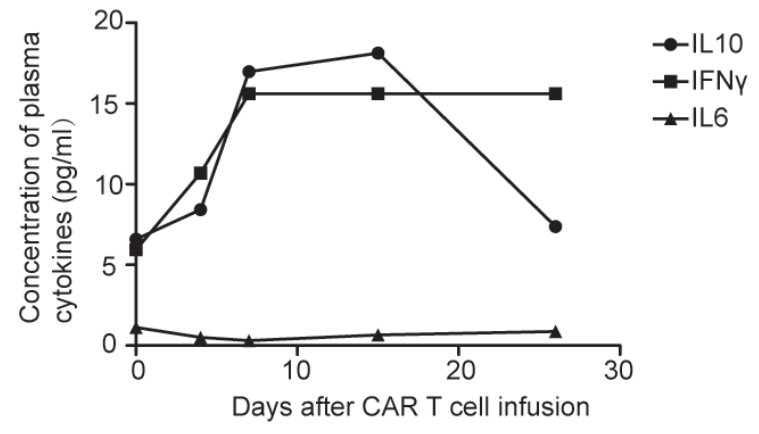

